http://dx.doi.org/10.18778/2196-8403.2009.09

\title{
HARTMUT STEINECKE
}

\section{Mit Lenau zwischen West und Ost}

W roku 1964 utworzone zostało w neutralnej Austrii „Międzynarodowe Towarzystwo Lenaua“. Na konferencjach tego towarzystwa spotykali się naukowcy z licznych państw leżących po obu stronach ,żelaznej kurtyny'. Praca nad historyczno-krytycznym wydaniem dzieł Lenaua doprowadziła do intensywnej współpracy germanistów z RFN, NRD, Austrii, Węgier i Polski. Artykuł jest relacją o trudach i sukcesach tego przekraczającego granice projektu.

1964 wurde im neutralen Österreich die „Internationale Lenau-Gesellschaft“ gegründet. Bei ihren Tagungen trafen sich Wissenschaftler aus zahlreichen Ländern diesseits und jenseits des ,Eisernen Vorhangs'. Die Arbeit an der historisch-kritischen LenauAusgabe führte zu einer intensiven Zusammenarbeit von Germanisten aus der Bundesrepublik Deutschland und der DDR, Österreich, Ungarn und Polen. Berichtet wird von den Mühen und den Erfolgen dieses Grenzen überschreitenden Projekts.

In 1964, in neutral Austria, the „International Lenau Society“ was established. Scholars from many countries on both sides of the ,Iron Curtain" met at the conferences of this society. Joint effort on the historical and critical publication of the works of Lenau led to intensive co-operation of Germanists from East and West Germany, Austria, Hungary and Poland. The article relates the difficulties and successes of that transborder project.

Nach dem Mauerbau 1961 und der Verschärfung der politischen Konfrontation zwischen West und Ost wurden die meisten der wenigen noch bestehenden Gemeinschaftsprojekte zwischen Wissenschaftlern von beiden Seiten des ,Eisernen Vorhangs“ abgebrochen. So war es ungewöhnlich, dass in diesen Jahren auch ein neues Kooperationsvorhaben begonnen und erfolgreich durchgeführt wurde: eine historisch-kritische Nikolaus-Lenau-Ausgabe, die Wissenschaftler aus der DDR, Ungarn und Polen einerseits, Österreich und der Bundesrepublik Deutschland andererseits zusammenführte. 
Hartmut Steinecke

Dazu bedurfte es allerdings besonderer Voraussetzungen, die wichtigsten waren kulturpolitischer Art. Die Biographie des Dichters aus der ersten Hälfte des 19. Jhd.s machte ihn zum idealen Gegenstand der Zusammenarbeit: geboren und aufgewachsen im habsburgischen Ungarn, späteres Leben in Mähren, Österreich und Deutschland. Die wichtigste politische Folgerung daraus war, dass die Initiative zu einer Kooperation von einer Gesellschaft im neutralen Österreich ausging und zunächst vor allem von Österreichern und Ungarn getragen wurde: der „Internationalen Lenau-Gesellschaft“, gegründet 1964 in Stockerau bei Wien (wo Lenau einige Lebensjahre verbrachte).

In der Satzung war als „Zweck der Gesellschaft“ nicht nur das bei einer Dichtervereinigung Erwartbare angegeben: ,die Pflege des Andenkens an Nikolaus Lenau, die Erforschung und Popularisierung seines Werkes“, sondern auch: „die Vertiefung der Völkerverständigung“. Dass dies nicht nur eines der üblichen allgemeinen Lippenbekenntnisse war, zeigten die bei der Gründungstagung gehaltenen Vorträge zweier hochrangiger österreichischen Politiker. Der Außenminister (und spätere langjährige Bundeskanzler) Dr. Bruno Kreisky sprach über „,Die österreichische Kunst und Kultur unserer Zeit - ein Beitrag zur Gestaltung einer friedlichen Welt“. Er forderte auf zur Selbstkritik Österreichs, zur Abwendung von einem selbstgefälligen Patriotismus, Hinwendung zur Weltaufgeschlossenheit, Förderung von internationalen Projekten als Beitrag des neutralen Österreich zur Weltkultur. In ähnlicher Weise stellte der Unterrichtsminister a.D. Dr. Heinrich Drimmel in seinem Vortrag mit dem Titel „Ost-West-Begegnung“ die politische Lage dar, die es als Österreichs Hauptaufgabe erscheinen lasse, als Mittler zwischen beiden Blöcken aufzutreten. Er forderte dazu auf, die seit Jahrhunderten gemeinsame Kultur und Literatur als Chance zu betrachten, die politischen Gegensätze zu überbrücken: „Hier um Wien besteht heute in der zweigeteilten Welt von Ost und West eine Brücke, eine der wenigen Brücken, die intakt sind.“

Dieser politische und kulturpolitische Kontext der Arbeit innerhalb der Gesellschaft blieb in den weiteren 25 Jahren der Spaltung Europas erhalten. Seit 1970 war der österreichische Bundesminister für Kultus und Unterricht Fred Sinowatz Präsident der ILG. (Später folgte er Kreisky als Bundeskanzler.) Diese personelle Konstellation öffnete natürlich zahlreiche Kontaktmöglichkeiten, die einer rein literarischen oder wissenschaftlichen Vereinigung verschlossen geblieben wären. Das traf in gleicher Weise auf den zweiten politischen Pfeiler der ILG zu: die Kommunen. Gründungsmitglieder waren eine Reihe von „Lenau-Städten“ in Österreich, Ungarn und Deutschland, die 
meisten Präsidenten bzw. geschäftsführenden Präsidenten der Gesellschaft waren die Bürgermeister Stockeraus, die Kommunen richteten die meisten Tagungen aus. Das schuf einen organisatorischen und finanziellen Rahmen, der die wissenschaftlichen Begegnungen erst ermöglichte. Für die Wissenschaftler aus den Ländern östlich des Eisernen Vorhangs wären sonst regelmäßige Besuche im westlichen Ausland weder finanziell möglich noch politisch erlaubt gewesen, wenn sie nicht zu den linientreuen Reisekadern gehört hätten. Ich selbst wurde - aufgrund zweier Publikationen über Lenau - 1971 erstmals zu einer Tagung der ILG eingeladen und war seitdem regelmäßiger Teilnehmer, bald auch aktiv im Wissenschaftlichen Beirat; ein Hauptmotiv für dieses Engagement war von Beginn an, dass man auf diese Weise sehr viel leichter als üblich in Länder des ,Ostblocks' reisen und mit Kollegen aus diesen Ländern zusammentreffen konnte.

Bereits bei der Gründung der ILG wurde als ein Fernziel eine neue LenauAusgabe genannt. Antal Mádl, Dozent in Budapest, war die treibende Kraft: Seit 1966 - 20 Jahre lang - war er Vorsitzender des Wissenschaftlichen Beirates, in den für die Ausgabe kritischen 70er Jahren Kulturattaché seines Landes in Wien, dann jahrzehntelang Direktor des bedeutendsten germanistischen Seminars in Ungarn, an der Eötvös Loránd Universität. Die ersten Entwürfe einer neuen Ausgabe - von Gerhart Baumann und Gerhard Neumann (Freiburg) - zielten auf eine Verbesserung der Ausgabe Eduard Castles von 1910-23. Walter Dietze (Leipzig) stellte 1967 vor der Gesellschaft seine im Entstehen begriffene, auf Castle beruhende „kritische Ausgabe“ vor (sie erschien 1970 in zwei Bänden, ohne Beteiligung der ILG). Herbert Zeman (Wien), Norbert Oellers (Bonn) und ich entwarfen 1974 den Plan einer völligen Neuausgabe. Auf verschiedenen Tagungen wurden die Möglichkeiten und Modelle diskutiert. Schließlich setzte sich die Einsicht in die Notwendigkeit einer neu zu erarbeitenden historisch-kritischen Ausgabe durch. Die drei Wissenschaftler aus Österreich und der Bundesrepublik stellten auf einer Tagung $1977 \mathrm{ihr}$ detailliertes Konzept vor, das einhellige Zustimmung fand. Der Ausrichtung der ILG folgend sollte diese Ausgabe in der Zusammensetzung ihrer Herausgeber die Internationalität der Gesellschaft spiegeln. So kristallisierte sich schließlich eine Gruppe von acht Wissenschaftlern heraus, die die insgesamt neun Bände betreuen sollten: Zeman und Mádl die beiden Gedichtbände, Hans-Georg Werner (Halle), Helmut Brandt (Jena) und Gerhart Koziełek (später: Kosellek, Wrocław/Breslau) die beiden Epenbände sowie András Vizkelety (Budapest), Oellers und Steinecke (Paderborn) die fünf Brief- und Prosabände (schließlich traten noch einige Mitarbeiter als Mitherausgeber hinzu). Bei der Tagung 1981 stellte der Präsident Sinowatz (zu 
dieser Zeit Vizekanzler Österreichs) die Bedeutung Lenaus und der ihm gewidmeten historisch-kritischen Ausgabe für die mitteleuropäische Kulturpolitik und Verständigung heraus: Lenau sei „im Gesamtraum der alten österreichischen Monarchie als der möglicherweise einzige Dichter zu nennen [...], den die Polen, die Tschechen und Slowaken, die Ungarn und die Rumänen, die Südslawen, die Deutschen und die Österreicher gleichermaßen gekannt, verehrt und geliebt haben. [...] In Lenaus Geist tritt unsere Gesellschaft zusammen, ein Werk zu schaffen, das gleichermaßen von allen genannten Völkern getragen wird. Ich meine eine historisch-kritische Gesamtausgabe der Werke und Briefe des Dichters.“

Derartiger Gemeinschaftsrethorik mit ihren Vorschusslorbeeren standen die Mühen der Ebenen der Konkretisierung des Projekts von Wissenschaftlern aus fünf Staaten gegenüber. Zu diesen pragmatischen Problemen des Alltags gehörte zum Beispiel die Bestimmung eines Gesamtherausgebers. Durch seine Position als Vorsitzender des Wissenschaftlichen Beirates hätte diese Rolle eigentlich Mádl zugestanden. Er wollte sie nicht übernehmen, da er sich nicht als Editionsfachmann ansah. Der ausgewiesenste Editor des Kreises, Norbert Oellers, fühlte sich mit der schwierigen Situation der SchillerNationalausgabe, eines deutsch-deutschen Gemeinschaftsprojekts, wissenschaftspolitisch mehr als ausgelastet. So einigte man sich zunächst darauf, Mádl und Zeman als eher organisatorisch zu verstehende „Koordinatoren“ zu benennen; später wuchs mir diese Rolle zu, als ich in der Nachfolge Mádls in den 80er Jahren zum Vorsitzenden des Wissenschaftlichen Beirates gewählt wurde.

Das Fehlen eines offiziellen Herausgebers machte alle Herausgeber gleich mit der Konsequenz, dass Helmut Brandt, der einen halben Band betreute, dank des Alphabets bibliographisch für die Ausgabe steht. Theoretisch unterwarfen sich alle Herausgeber Mehrheitsentscheidungen - auch für demokratieerprobte Wissenschaftler der Bundesrepublik Deutschland und Österreichs eine etwas abenteuerliche Vorstellung. Meistens diskutierten wir so lange, bis sich die Argumente der Herausgeber mit der größten Editionserfahrung durchsetzten. Schwierig wurde es bereits bei terminologischen Fragen, z.B. wie Jahreszahlen angegeben werden sollten (v. d.Z. oder v. Chr.) oder wie das Herausgeberensemble offiziell firmieren sollte. Im Entwurf der DDRKollegen war ganz unbefangen von einem „Herausgeberkollektiv“ die Rede; nach einer kontroversen Diskussion mit politischen Untertönen wurde der Punkt zurückgestellt; zwei Stunden und mehrere Gläser österreichischen Weins später wurde der Kasus nochmals aufgegriffen, nun mit überwiegend 
ironischen Akzenten und der einvernehmlichen Entscheidung für „Herausgeberkollegium" beendet.

Schwieriger als derlei Geplänkel war die Festlegung von editorischen Richtlinien, denn es gab nicht nur sehr unterschiedliche wissenschaftsgeschichtlich bedingte Vorstellungen von den Aufgaben einer historisch-kritischen Ausgabe (so war nach ,westlicher“ Definition Dietzes „kritische Ausgabe“ keineswegs „kritisch“), auch die wissenschaftspolitischen Aspekte waren durchaus unterschiedlich. In den östlichen Ländern waren editorische Großprojekte im Allgemeinen den Akademien der Wissenschaft vorbehalten, für die meisten Schriftsteller galten (nicht zuletzt aus finanziellen Gründen) Studienausgaben als völlig ausreichend; und selbst wenn man höchstes editorisches Niveau anstrebte, blieben große Differenzen zu ,westlichen 'Vorstellungen - das hat das bekannte Schicksal der zunächst als "gesamtdeutsch“ geplanten historisch-kritischen Heine-Ausgabe gezeigt, die sich nach 1961 zum scharfen Gegeneinander der Editoren und der wissenschaftsgeschichtlich bisher einmaligen Kuriosität zweier gleichzeitig entstehender konkurrierender historisch-kritischer Ausgaben entwickelte.

Auch wenn die beteiligten Wissenschaftler bereit waren, überzeugende Argumente zu akzeptieren, standen sie doch auch unter dem Druck ihrer jeweiligen heimischen Wissenschaftsorganisationen oder - etwa in der DDR - deren vorgesetzten Ministerien. Das galt im Grunde auch für die Herausgeber der Bundesrepublik, denn die DFG hätte kein Projekt gefördert, das unterhalb der Schwelle der üblichen Standards historisch-kritischer Ausgaben lag. Schließlich konnten die Teilnehmer aus Ungarn, Polen und der DDR durchsetzen, dass ihnen die Mitarbeit an einem Projekt genehmigt wurde, das diese Standards verbindlich machte. So wurden die erforderlichen Mitarbeiter in der Bundesrepublik Deutschland von der DFG sowie dem Beauftragten der Bundesregierung für Angelegenheiten der Kultur und der Medien gefördert, in Österreich von dem Ludwig Boltzmann-Institut sowie der wissenschaftlichen Landesakademie für Niederösterreich, in Ungarn von der Akademie der Wissenschaften sowie in der DDR vom Ministerium für Hoch- und Fachschulwesen. Die zentralen Kosten, vor allem für die Archivreisen und die Druckkostenzuschüsse, wurden weitgehend vom Bundesministerium für Unterricht und Kunst Wien, daneben auch von verschiedenen Lenau-Städten getragen. Nach Klärung all dieser Fragen wurden 1982 die Verträge unterschrieben; da die Bände zeitlich weitgehend nebeneinander erarbeitet wurden, gingen wir zuversichtlich von einem Erscheinen der gesamten Ausgabe binnen 8-10 Jahren aus. 
Die zahlreichen Treffen im Rahmen der Jahrestagungen der ILG sowie bei separaten Herausgebertreffen in den verschiedenen Ländern führten bei fast allen beteiligten Wissenschaftlern nach einiger Zeit zu einer großen Offenheit, allerdings eher unter vier oder auch sechs bis acht Augen als in der, großen Runde'. Die Kollegen aus dem Osten machten keinen Hehl daraus, dass sie - insbesondere in der Anfangsphase des Unternehmens - über ihre Reisen und die Treffen zu Hause berichten mussten, aber sie legten auch dar, was sie berichten würden. Und sehr offen wurde über die unterschiedlichen Motive gesprochen: Für die Kollegen der DDR stand die Möglichkeit, in das „NSA“ (nichtsozialistische Ausland) zu reisen - nach einiger Zeit sogar ohne ,Begleiter ${ }^{6}$, zu Treffen und zur Archivarbeit, die ebenso in Westdevisen finanziert wurden wie die zu erwartenden Honorare, eine wesentliche Rolle; für Norbert Oellers und mich waren umgekehrt die Möglichkeiten, verschiedene Länder und Kollegen des Ostblocks näher und ungehinderter als üblich kennenzulernen, starke Argumente für eine Mitarbeit. Intensive Beziehungen ergaben sich mit allen Beteiligten: Wir luden uns auch untereinander ein, zu Vorträgen, Teilnahme an Tagungen unserer Universitäten, es kam zu Kooperationen bei weiteren Projekten, die mit Lenau nichts zu tun hatten. Das galt für Norbert Oellers und mich in besonderer Weise für den Umgang mit HansGeorg Werner und Helmut Brandt aus der DDR, die bald zu Freunden wurden, sowie für die Beziehungen zu den ungarischen Kollegen. Hier führten die zahlreichen intensiven Kontakte bereits in den 70er Jahren zu einem intensiven Austausch, der schließlich in einer offiziellen Partnerschaft zwischen den Germanistischen Instituten der Universitäten Budapest und Paderborn mündete. Da Budapest eine Partnerschaft bereits länger und früher mit der Universität Jena verband, erleichterte dies die Gewinnung eines Herausgebers von der dortigen Universität, des damaligen Dozenten Helmut Brandt.

Durch diesen engen Kontakt erhielten wir ,Westler' auch einen gewissen Einblick in die innenpolitischen Entwicklungen in Ungarn, Polen und der DDR. Die Jahrestagung 1989 - zum 25-jährigen Bestehen der ILG - begann in Mosonmagyaróvár (Ungarn) und wurde in Stockerau (Österreich) fortgesetzt, keine $50 \mathrm{~km}$ voneinander entfernt, noch immer getrennt durch den ,Eisernen Vorhang', der allerdings in den zurückliegenden Wochen gerade hier, an der ungarisch-österreichischen Grenze, bereits löcherig geworden war. Alle Teilnehmer fuhren also von Ungarn nach Österreich, mit Bussen oder Privatwagen. Wir nahmen die DDR-Kollegen im Auto mit. An der Grenze wurden wir von den ungarischen Beamten, deren geschulter Blick bekanntlich sofort das Herkunftsland eines Deutschen erkannte, verständnisinnig grinsend, freundlich durchgewinkt. Zur Verblüffung der Zöllner stoppten 
wir, weil die DDR-Bürger einen Ausreisestempel benötigten, da sie ja wieder zurückreisen wollten - im August 1989 ein höchst seltener und seltsamer, aber in diesem Fall verständlicher Wunsch.

Das, was in diesen Tagen zu sehen und von unseren Kollegen zu hören war, fasste ich in meiner Eröffnungsansprache der Jahrestagung, bei der auch die beiden ersten Bände der historisch-kritischen Ausgabe der Öffentlichkeit vorgestellt wurden, zusammen. Ich sagte seinerzeit, am 30. August 1989:

[Die ILG] trifft sich seit einem Vierteljahrhundert unter dem Namen Lenaus als Symbol eines Nationen übergreifenden Kulturverständnisses. Wir alle hofften, daß die ILG damit zu einem der Vorläufer gesamtgesellschaftlicher und politischer Prozesse würde. Es ist überaus erfreulich, am Beispiel unseres Gastgeberlandes Ungarn zu erleben, wie eine solche Hoffnung, eine solche Vision Schritt um Schritt und zuletzt geradezu sprunghaft Wirklichkeit wird. Dies verstärkt meine Hoffnung, daß dieser Weg auch für die Länder möglich wird, für die er bisher noch als Utopie gilt. Da Kunst uns lehrt, das Undenkbare zu denken: Warum sollten wir uns nicht ausmalen, daß nicht nur die Herausgeber der Lenau-Ausgabe aus Österreich, Ungarn und der Bundesrepublik Deutschland, sondern auch die aus der DDR und aus Polen den Abschluß der Ausgabe 1994 bei einer Tagung feiern, zu der sie ohne Visa und Ausreisegenehmigung anreisten? Und daß dies auch im Laufe der neunziger Jahre für unsere Referenten und Teilnehmer aus der ČSSR und Rumänien, Bulgarien und der Sowjetunion gilt?

Die politische Vision erfüllte sich rascher und weitergehend als vorausgeahnt, paradoxerweise verhinderte sie allerdings gerade, dass die andere $\mathrm{Zu}$ kunftsprognose - den Abschluss der Ausgabe betreffend - erreicht werden konnte. Die neuen Freiheiten, die sich die Menschen in Ungarn, Polen und der DDR erkämpft hatten, führten fast allenthalben auch zu größeren beruflichen Problemen durch eine tiefgreifende Umstellung der Systeme und durch interne Probleme an den Instituten (wobei auch einige der Kollegen, die dank der ILG eine gewisse Reisefreiheit genossen, verschiedentlich dem Neid der Kollegen ausgesetzt waren und dem Verdacht, dass dies durch Nähe zu den staatlichen Organen bedingt gewesen sein könnte - die westlichen Partner konnten hierbei gelegentlich zu Zeugen der Integrität werden). Während die Bände der Herausgeber aus der Bundesrepublik bis 1993 vorlagen, folgten die Bände mit dem österreichischen und ungarischen Herausgeber 1995, die der Mitherausgeber aus der DDR und Polen 1997 und 2004.

Die engen, vor der Wende geknüpften Beziehungen bewährten sich auch in den nächsten Jahren. Ich selbst wurde als erster Gastprofessor nach der Wende im Frühjahr 1990 für ein Semester an die Eötvös Loránd-Universität Budapest berufen. Unsere Zusammenarbeit erfasst mittlerweile längst die nächste Generation und ist in europäische Forschungszusammenhänge über- 
gegangen. Junge Wissenschaftler, die wir als Doktoranden oder Mitarbeiter zu den Tagungen der ILG eingeladen oder in die Editionsarbeit eingebunden hatten, führten und führen - inzwischen etabliert - die Tradition der Zusammenarbeit fort, so in verschiedenen gemeinsamen Tagungen der Germanistischen Institute von Paderborn mit Budapest sowie - im Mai 2008 - mit Wrocław.

Das gesamte Werk Lenaus umfasst weniger als 2.000 Textseiten. Wissenschaftsökonomisch war die Beteiligung von acht Wissenschaftlern aus fünf Nationen eher unsinnig: Die drei Germanisten aus Österreich und der Bundesrepublik hätten die Ausgabe sicher in wesentlich kürzerer Zeit in (mindestens) gleicher Qualität erstellen können. Doch nie haben wir die internationale Ausweitung des Herausgebergremiums bedauert. Sie war für alle Beteiligten ein Gewinn, wenn dieser auch für jeden Einzelnen anders aussah. Gemeinsam gelang es, in einer Zeit der extremen politischen Gegensätze in einem kleinen wissenschafts- und kulturpolitischen Bereich einige Verbindungsfäden zu ziehen zwischen den beiden deutschen Staaten und zwischen verschiedenen Ländern auf beiden Seiten der Grenze durch Europa. 